\title{
Nursing and midwifery students will be left behind
}

Nurses and midwives are being celebrated worldwide as heroes. Frontline nurses and midwives are being credited for their tireless efforts in managing the COVID-19 pandemic, including the associated positive health outcomes. However, this pandemic has exposed the continued marginalisation of nursing and midwifery students throughout Africa.

According to the World Economic Forum (2020), higher education institutions in Africa struggled to adapt their planned academic activities to disruptions associated with the COVID-19 pandemic. At the beginning of the pandemic, the majority of these institutions suspended their academic activities, while only a handful resorted to emergency remote teaching as a strategy for continued academic activities. In some countries, higher education institutions received additional support from governments to buffer expenses associated with remote learning and teaching. Computers, zero-rated online access, and students receiving data from their institutions are examples of support rendered to students to enable access to learning materials in remote locations. In South Africa, this support was aimed at 'not leaving any student behind' and salvaging the academic year through continued learning in higher education institutions.

Unfortunately, nursing and midwifery students in Africa will be left behind because their training predominantly occurs in hospital-based nursing education institutions governed by the ministries of health. ${ }^{[1]}$ This situation is a result of the complex historical legacies aligned with the establishment of nursing education in Africa. These nursing education institutions are hospital departments and not recognised as part of higher education. They compete with other hospital departments for the finite hospital budget, and in crises similar to the COVID-19 pandemic, they are not prioritised. Consequently, at the beginning of the pandemic, planned academic activities for nursing and midwifery students in Africa were suspended. The clinical platform could not admit these students owing to limitations in personal protective equipment. Furthermore, while at home, the students did not receive any additional resources or support for continued learning.

With the general improvement in health outcomes and continued easing of COVID-19-related restrictions in most African countries, higher education institutions are working towards the reopening of academic activities. These institutions are expected to ensure the safety of students and staff by meeting stricter regulations related to physical distancing, sanitisation, health screening and personal protective equipment. Governments are working directly with these institutions by supporting risk-adjusted strategies and resource allocation. Nursing education institutions will fail to meet stricter COVID-19-related regulations, as budgets within ministries of health prioritise patient care. The clinical platform is not ready for students, furthering their plight in meeting regulatory requirements. Nursing and midwifery students will continue being at home and not being engaged in any form of learning, unlike their counterparts in higher education. The consequences of leaving nursing and midwifery students behind will have a ripple effect on the already fragile healthcare system.

COVID-19-related experiences have exhumed discussions around the governance of nursing education in Africa. Questions on the appropriateness of health ministries governing nursing education have been discussed in the literature, with various authors already calling for the migration of the governance of nursing education to higher education. ${ }^{[2,3]}$ Armstrong and Rispel ${ }^{[4]}$ in their argument for the professionalisation of nursing, reflect on the need for nursing and midwifery qualifications to be on par with other higher education qualifications, articulating through similar qualification frameworks. Consequently, nursing education institutions will be expected to meet quality-assurance standards set for all higher education institutions, while their students may benefit from all risk-adjusted strategies aimed at continued learning in crises. However, migrating the governance of nursing and midwifery education to higher education may have crippling consequences for the healthcare system, which are related to budgets and human resources, as students are often perceived as part of the staff. ${ }^{[5]}$ This delicate situation needs renewed discussion among all nursing education stakeholders, aimed at a sustainable solution that prioritises learning and the end of the continued marginalisation of nursing and midwifery students.

At the beginning of 2020, we called for manuscripts for the special focus issue on nursing and midwifery education in Africa. This call received an overwhelming response from authors across the continent, presenting work done in undergraduate, postgraduate and continuing education for nurses and midwives. The African Journal of Health Professions Education has dedicated the last two issues of this volume to the special focus issue.

\section{Champion N Nyoni}

Guest editor: Special focus issue on nursing and midwifery education, School of Nursing, Faculty of Health Sciences, University of the Free State, Bloemfontein, South Africa nyonic@ufs.ac.za

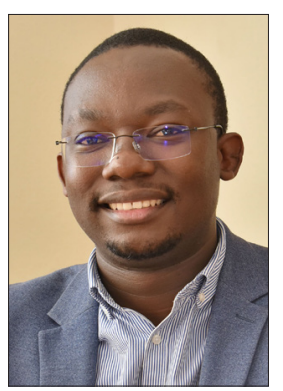

1. Blaauw D, Ditlopo P, Rispel LC. Nursing education reform in South Africa - lessons from a policy analysis stud Glob Health Action 2014;7(1):26401. https://doi.org/10.3402/gha.v7.26401

2. Rispel L, Schneider H. Professionalization of South African nursing: Who benefits? Int J Health Serv 1991;21(1):109-126. https://doi.org/10.2190/GUHD-GWFQ-YWJJ-EYKH

3. Botma Y. Implications of accreditation criteria when transforming a traditional nursing curriculum to a competency-based curriculum. Int J Africa Nurs Sci 2014;1:23-28. https://doi.org/10.1016/j.ijans. 2014.06 .002

4. Armstrong SJ, Rispel LC. Social accountability and nursing education in South Africa. Glob Health Action 4. Armstrong SJ, Rispel LC. Social accountability and n
2015;8(1):27879. https://doi.org/10.3402/gha.v8.27879

5. Bvumbwe T, Mtshali N. Nursing education challenges and solutions in Sub Saharan Africa: An integrative review BMC Nurs 2018;17(1):3. https://doi.org/10.1186/s12912-018-0272-4

Afr J Health Professions Educ 2020;12(3):94. https://doi.org/10.7196/AJHPE.2020.v12i3.1441 